\title{
High precision capacitance displacement measurement circuit design
}

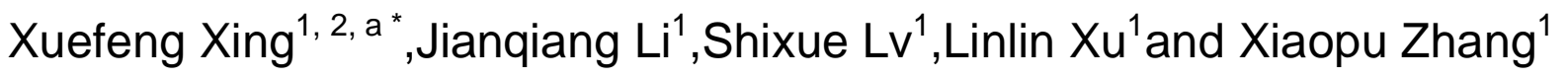 \\ ${ }^{1}$ College of Instrumentation and Electrical Engineering, Jilin University, Changchun, P. R. China \\ ${ }^{2}$ National Instrument Engineering Technology Research Center of geophysical exploration, Jilin \\ University, Changchun, P. R. China \\ axing@jlu.edu.cn
}

Keywords:high precision displacement measurement, analog circuit, micro controller

Abstract:A high resolution digital integrated chip capacitor measurement system was designed.The major components of the system mainly includedmain control chip, capacitance transducer, data storage and communication interface.ARM7-based mcro controllerSTM32F207was used as the main control chip, capacitance measurement chip AD7747produced by AD Company with sensitivity can be up to 20 af was used as capacitance transducer. The capacitance measurement range ofAD 7747 is -8 pf to +8 pf, the maximum common-mode immutable capacitance can reach 17 pf anddata update rate range is between $5 \mathrm{~Hz}$ to $45 \mathrm{~Hz}$. The absolute error of test circuit was calculated by the fixed capacity test and error analysis. The final results were obtained after subtracting the absolute error, and the final test error is less than $3 \%$.

\section{Introduction}

With the high-speed development of modern science and technology, the requirement for theaccuracy of the instrument processinghas been increased. Nowadays, precision machining technologyhas become an important index of the development of industry and science technology ${ }^{[1-4]}$. The ultra-precision measurement technology is always explored andgained more attention. The precision measurement technique is the key part of the measurement of machinery industry development in almost all developed countries.Capacitive displacement sensor technology is themost mainly used measurement method. This technology obtained more attention and has been applied in many different fields.

Currently, commonlyuseddisplacementmeasurement sensor mainly included capacitive and inductive displacement sensor and optical fiber displacement sensor. These different sensors have their own advantages and disadvantages and can be used in various occasion ${ }^{[5-7]}$. The capacitance displacement measurement sensor chosen here has many advantagescompared withothercapacitive displacement sensors: (1) the dynamic response is sensitive. (2) the structure is simple. (3) stable enough at high temperature. Most capacitive sensorsuse air as a dielectric, the problem of self-heating isnegligible. Therefore, the stability is guaranteed under the stable-temperatureenvironment. (4)the amount of change is significant.(5)non-contact measurement is feasible. The internal friction exists in resistive and optical fibersensor because of the mechanicalparts. This kind of deviation is always difficult to eliminate, so the structure of capacitive displacement measurement has been widely used.

Plate capacitive displacement sensor is the most basicsensor, it measures by converting the value of the capacitance to measure displacement. The framework of unipolar plate capacitive sensor was shown in Figure 1. 


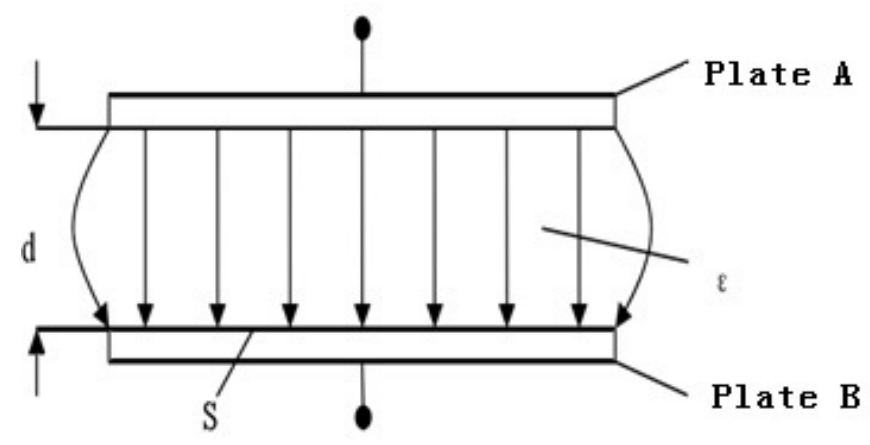

Figure 1.The framework of unipolar plate capacitive sensor

The coefficient of capacitive sensors can be adjust and four basic parameters can be changed.Capacitance between the two plates is $\quad-. s$ is the plate effective area.dis the plate spacing. $s$ is the plate medium between the dielectric constant.

\section{The hardware circuit design}

Mainly parts ofmeasurement system included: main control chip, capacitance transducer, data storage and communication interface.The main control chip using micro controllerSTM32F207 based onARM7, capacitance transducer using the capacitance measurement chip AD7747produced byAD Company.AD7747has many advantages such as the height that other chips can not reach. AD7747can take advantageof the single chip solutionand can interface with single sensor, the resolutionis relatively high. The system structurewas shown in Figure 2.

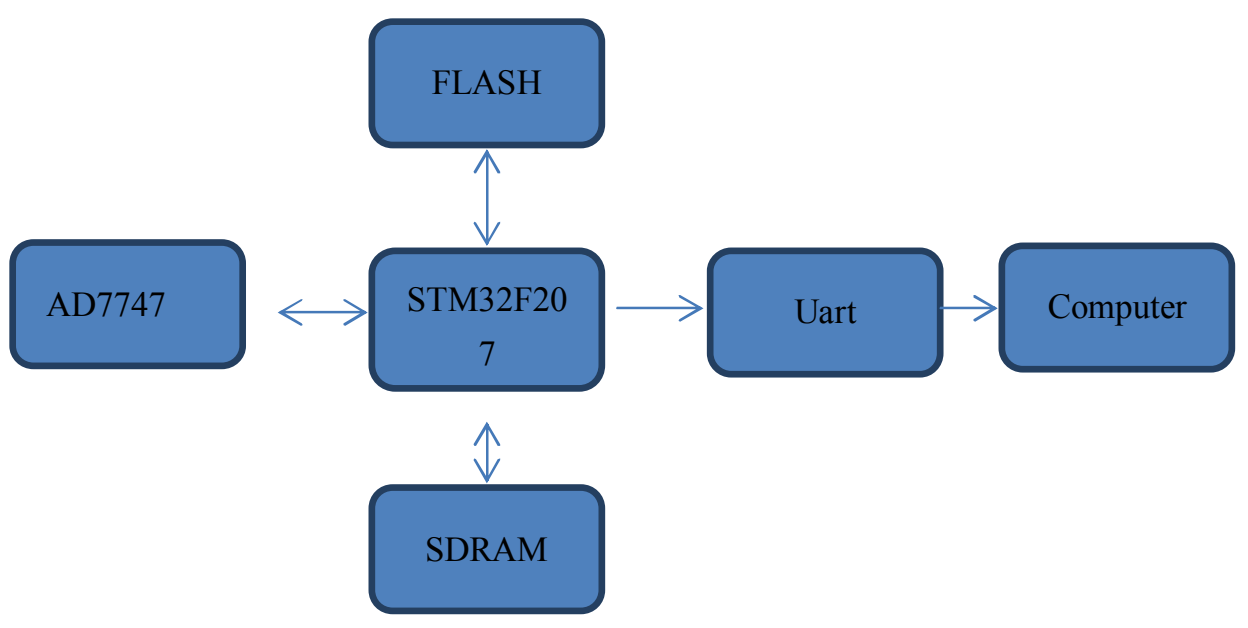

Figure2. System structure diagram

The minimum resolution of AD7747 can reach 20 af. The linearity and precision of the AD7747 can reach $0.01 \%$ and $10 \mathrm{ff}$. AD7747 capacitance measurement range isfrom- 8 pf to 8 pf.Its maximum common-mode immutable capacitance can reach 17 pf. AD7747 update rate range isfrom $5 \mathrm{HZ}$ to $45 \mathrm{HZ}$.AD7747 is not only a kind of active shielding to protect sensor, or a piece of internal temperature sensor, the piece of temperature sensor in its resolution can reach $0.1{ }^{\circ} \mathrm{C}$, precision can reach $2^{\circ} \mathrm{C}, \mathrm{AD} 7747$ is roughly divided into three parts, including voltage input channel part, 
two-wire serial interface parts, i.e. I2C part and internal clock generator parts. The working voltageof AD7747 isfrom $2.7 \mathrm{~V}$ to $5.25 \mathrm{~V}$.

Data memory has a nonvolatile memory FLASH and high-speed power loss of SDRAM.These components were used to store the STM32 configuration information, long-term preserve information and obtaindata from the high-speed data acquisition.Generic 232 interface Uartwas used as the communication structure to swap datawith the computer.

Herein,STM32F207 was selected as the capacitance displacement digital master control chip of the circuit.STM32 was selectedas main control chip and the I2C signal module, power supply and the clock module, serial communication module, a button control module and PC display moduleconstitute the capacitance displacement measurement circuit of the control module of hardware system. The acquisition and transmission were achieved by the upper machine controller.

\section{Fixed capacitor test}

The design of acquisition system for fixed capacitor was tested, and obtainedthe accurate absolute error and the correcting error. The difference variable capacitor test got no results due to the uncertainty of the differential capacitance.Table 1 shown a record of the average values of 20timesmeasurements for each item. Theabsolute error is $0.222 \mathrm{pf}$, and the measurement error is less than $3 \%$. 
Table 1 . The average values of 20timesmeasurements

\begin{tabular}{|l|l|l|l|l|l|}
\hline $\begin{array}{l}\text { Standard } \\
\text { capacitance(pf) }\end{array}$ & $\begin{array}{l}\text { Measured } \\
\text { value(pf) }\end{array}$ & error & $\begin{array}{l}\text { Standard } \\
\text { capacitance(pf) }\end{array}$ & $\begin{array}{l}\text { Measured } \\
\text { value(pf) }\end{array}$ & error \\
\hline 3.3 & 3.365 & 0.06 & 3.3 & 3.290 & 0.01 \\
\hline 3.3 & 3.129 & 0.17 & 4.3 & 4.323 & 0.02 \\
\hline 4.3 & 3.992 & 0.30 & 4.3 & 4.582 & 0.28 \\
\hline 7.0 & 7.161 & 0.16 & 7.0 & 6.581 & 0.41 \\
\hline 7.0 & 7.285 & 0.28 & 8.2 & 8.148 & 0.05 \\
\hline 8.2 & 7.934 & 0.26 & 8.2 & 8.474 & 0.27 \\
\hline 15.0 & 15.017 & 0.01 & 15.0 & 15.092 & 0.09 \\
\hline 15.0 & 14.801 & 0.19 & 5.0 & 5.220 & 0.22 \\
\hline
\end{tabular}

\section{Conclusion}

ARM7-based micro controllerSTM32F207 was used asthe capacitance displacement digital master control chip. The measurement results were consistent with the standard values after subtracting the absolute error.The AD7747 was used to measure the differential capacitance and the satisfactory results were obtained.The differential capacitance plate of the linear range is too low, since the AD7747 is very easy to enter the saturated zone output, sothere is higher demand for thedesign of differential capacitance..

\section{References}

[1] J. Mazur, and Z. Swietach Efficient DOA estimation using tri-axial geophone, 2012 International Conference on Signals and Electronic Systems (Icses), 2012

[2] C. Stotter, and E. Angerer Evaluation of 3C microelectromechanical system data on a 2D line: Direct comparison with conventional vertical-component geophone arrays and PS-wave analysis, Geophysics, 2011, 76, (3), pp. B93-B101

[3] V.V. Reddy, V. Divya, A.W.H. Khong, and B.P. Ng Footstep Detection and Denoising using a Single Triaxial Geophone, Proceedings of the 2010 Ieee Asia Pacific Conference on Circuit and System (Apccas), 2010, pp. 1171-1174 [4] C.Y. Ding, A.A.H. Damen, and P.P.J. van den Bosch Robust Vibration Isolation by Frequency-Shaped Sliding Surface Control with Geophone Dynamics, Ieee Ind Elec, 2010

[5] X. Roset, M. Nogueras, A. Garcia, J. del Rio, and D. Sarria Geophone Calibration by means of hyperbaric chamber, Oceans 2009 - Europe, Vols 1 and 2, 2009, pp. 263-266

[6] A. Strollo, D. Bindi, S. Parolai, and K.H. Jackel On the suitability of $1 \mathrm{~s}$ geophone for ambient noise measurements in the $0.1-20 \mathrm{~Hz}$ frequency range: experimental outcomes, B Earthq Eng, 2008, 6, (1), pp. 141-147

[7] C.Z. Lv, D. Fan, A.Y. Wei, and Z.F. Yin A design of Geophone Tester Based on FPGA, 2008 Ieee International Conference on Automation and Logistics, Vols 1-6, 2008, pp. 587-590 